\title{
Therapeutic effect of mesenchymal stem cell therapy in the LVEF, LVEDV, and LVESV after myocardial infarction
}

\author{
Rabiei MM ${ }^{1,4}$, Khoramgah MS²,3, Darazam IA ${ }^{4}$, Vafaei-Nezhad S , Dargahi S ${ }^{3}$, Abbaszadeh HA ${ }^{2,3,1}$ \\ Department of Biology and Anatomical sciences, school of medicine, Shahid Beheshti University \\ of Medical Sciences, Tehran, Iran. Dr.abbaszadeh@sbmu.ac.ir
}

\section{ABSTRACT}

OBJECTIVES: The present study was aimed to investigate the therapeutic effect of mesenchymal Stem Cell Therapy in the left ventricular ejection fraction (LVEF), left ventricular enddiastolic volume (LVEDV), and left ventricular endsystolic volume (LVESV) after myocardial infarction (MI).

BACKGROUND: Previous investigations propose that stem cell therapy may help treat myocardial infarction (MI). However, there are controversial data from different studies.

METHODS: We studied the relevant scientific literature available up to 2020. Comprehensive Meta-Analysis Software (CMAS) Version 2.0 were used for statistical analyses. Fixed or random-effect model was used to identify the weighted mean difference (WMD) with 95\% confidence intervals (Cl). The statistically significant level used for interpreting publication bias was less than 0.05 .

RESULTS: We identified 30 studies that met the inclusion criteria. In the overall pooled estimate, cell therapy had an effect on the LVEF change from baseline to follow-up (WMD: $2.98 \mathrm{~mL}, 95 \% \mathrm{Cl}$ : 1.66 to 4.29 ). The pooled WMD was found to be -4.16 ( $95 \% \mathrm{Cl}:-7.91$ to -0.40 ) and -5.62 (95\% Cl: -9.20 to -2.00$)$, for LVEDV, and LVESV, respectively. Thus, reduction in LVEDV and LVESV were significant in the treatment group participants. CONCLUSIONS: The present systematic review indicated that cell therapy in patients, who have MI could be effective and applicable clinically (Tab. 3, Fig. 7, Ref. 48). Text in PDF www.elis.sk KEY WORDS: myocardial infarction, stem cell, systematic review, randomized clinical trials.

\section{Introduction}

Heart disease is still one of the main universal reasons of death and according to evidence it will increase in further years (1). It was estimated that 2.8 fold increase in death number due to heart disease would occur from 2000 to 2050 (2). The major problem in the people, who suffer from heart disease, is functional impairment, which is caused by ischemic failure (3). The key management of ischemic heart disease (IHD), which manifests

${ }^{1}$ Department of Biology and Anatomical sciences, school of medicine, Shahid Beheshti University of Medical Sciences, Tehran, Iran, ${ }^{2}$ Laser Application in Medical Sciences Research Center, Shahid Beheshti University of Medical Sciences, Tehran, Iran, ${ }^{3}$ Hearing disorders research center, Loghman hakim hospital, Shahid Beheshti University of Medical Sciences, Tehran, Iran, ${ }^{4}$ Infectious Diseases and Tropical Medicine Research Center, Shahid Beheshti University of Medical Sciences, Tehran, Iran, and ${ }^{5}$ Department of Anatomical Sciences, Faculty of Medicine, Birjand University of Medical Sciences, Birjand, Iran

Address for correspondence: H.A. Abbaszadeh, Dr, Laser Application in Medical Sciences Research Center, Shahid Beheshti University of Medical Sciences and Biology and Anatomical Sciences Department, School of Medicine, Shahid Beheshti University of Medical Sciences, Velenjak, Tehran 1985717443, Iran. PO Box: 19395-4719

Phone: +98.21.23872555, Fax: +98.21.22439976

Acknowledgment: We are thankful for the funding provided by Laser Application in Medical Sciences Research Center, Shahid Beheshti University of Medical Sciences, Tehran, Iran. clinically as MI and ischemic cardiomyopathy, is the effective integration of pharmacological therapy combined with modern reperfusion strategies that has improved the patients' long-term prognosis and raised their life expectancy (1). Although these current therapies declined the mortality rate after an acute MI, they were unable to recover infracted areas so the existence of damaged cardiomyocytes remains as an important risk factor for further progression to heart failure (4). Thus, triggering cardiomyocytes regeneration in damaged tissue could be the best way to recover the heart function and increase the affected people survival rate. In recent years, several investigations worked on myocardial regeneration through stem/progenitor cell transplantation therapy (5). Five kinds of stem cells containing bone marrow-derived stem cells (BMSCs) $(6,7)$ or bone marrow-derived mononuclear stem cells (BMMNCs), mesenchymal stem cells (MSC) (8), cardiac stem cells (CSCs), cardiosphere-derived stem cells (CDCs), and embryonic stem cells (ESCs) have mostly been studied for cardiomyocyte regeneration. The preclinical studies and some clinical studies suggested that stem cell therapy had a satisfactory effect on the performance of the cardiac function after heart failure (5). To determine the efficacy of novel treatments in heart failure, scientists usually measure important markers related to left ventricle because this chamber is in charge of pushing the blood into all the body and the existence of any failure on it could cause a variety of disabilities in the patient. Thus, most of clinical trials with the aim of assessing the efficiency of stem cells were 
Tab. S1. Search strategy for the PubMed/Medline database.

\begin{tabular}{ll}
\hline Search & Query \\
\hline$\# 3$ & $((((((((((((((($ Myocardial Infarction(MeSH Terms)) OR (Myocardial Infarction(Title/Abstract))) OR (myocardial failure(Title/Abstract))) \\
& OR (Myocardial Ischemia(Title/Abstract)))) OR (cardiomyopathy(Title/Abstract))) OR (myocardiopathy(Title/Abstract))) OR (Cardio- \\
& vascular Stroke(Title/Abstract))) OR (Myocardial Infarct(Title/Abstract))) OR (Heart Attack(Title/Abstract))) OR (cardiac failure(Title/ \\
& Abstract))) OR (heart failure(Title/Abstract)) AND ((((((Stem Cells(MeSH Terms)) OR (Stem Cells(Title/Abstract))) OR (mesenchy- \\
& mal stem cell(Title/Abstract))) OR (mesenchymal stromal cell(Title/Abstract))) OR (mesenchymal progenitor cell(Title/Abstract))) OR \\
& $($ Mesenchymal Stem Cells(MeSH Terms)) \\
\hline \#2 & ((((((((((((((Myocardial Infarction(MeSH Terms)) OR (Myocardial Infarction(Title/Abstract))) OR (myocardial failure(Title/Abstract))) \\
& OR (Myocardial Ischemia(Title/Abstract)))) OR (cardiomyopathy(Title/Abstract))) OR (myocardiopathy(Title/Abstract))) OR (Cardio- \\
& vascular Stroke(Title/Abstract))) OR (Myocardial Infarct(Title/Abstract))) OR (Heart Attack(Title/Abstract))) OR (cardiac failure(Title/ \\
& Abstract))) OR (heart failure(Title/Abstract)) \\
\hline \#1 & (((((Stem Cells(MeSH Terms)) OR (Stem Cells(Title/Abstract))) OR (mesenchymal stem cell(Title/Abstract))) OR (mesenchymal stromal \\
& cell(Title/Abstract))) OR (mesenchymal progenitor cell(Title/Abstract))) OR (Mesenchymal Stem Cells(MeSH Terms)) \\
\hline
\end{tabular}

investigated on left ventricular (LV) remodeling after treatment. In this way, watching on three measurable values, including left ventricular ejection fraction (LVEF), left ventricular end-systolic volumes (LVESV) from the baseline, and left ventricular enddiastolic volume (LVEDV) are more important (9-11). Whereas LVEF is an important marker to identify the extent of ventricles abnormalities after a heart disease, it is considered as a guide to estimate the risk of morbidity rate and also the treatment efficacy (9). As well, it has been demonstrated that measuring the LVESV could be the key marker to estimate the patient survival after MI (10). Another important factor that serves as a measurable value to demonstrate how well is the heart performing, is LVEDV (11). So, evaluating these three markers will provide a vision for scientists to find the outcome of novel treatment. Although preclinical and clinical research suggested that stem cell therapy has been safe and useful, there were some reports, which propound the idea that stem cell therapy are unable to treat the heart failure effectively. Thus, current study aimed to review the studies to evaluate the useful effects of stem cell therapy after MI through a comprehensive systematic review and meta-analysis.

\section{Material and methods}

This study is in fulfilment of the "Preferred Reporting Items for Systematic Reviews and Meta-Analyses" (PRISMA) statement (12).

\section{Search strategy and selection criteria}

We investigated the PubMed/Medline, EMBASE, Cochrane Library, Scopus, google scholar and reference lists of relevant articles from January 1, 2000 to January 1, 2020. The following keywords were used: myocardial infarction, myocar- dial failure, stem cells, and mesenchymal stem cell. The search was restricted to English articles. Details of strategies used in PubMed/Medline are given in the Table S1 in Supplement. Only randomized controlled trials (RCT) studies, in which stem cells were used as a treatment for MI, were included. RCTs with the following criteria were included: (1) the intervention involved any stem cells without a limitation by administration route or dose, (2) at least three months' follow-up period, and (3) in the control arm patients did not receive stem cells. Review articles, duplicate publications, and articles with unclear data were ruled out from the analysis.

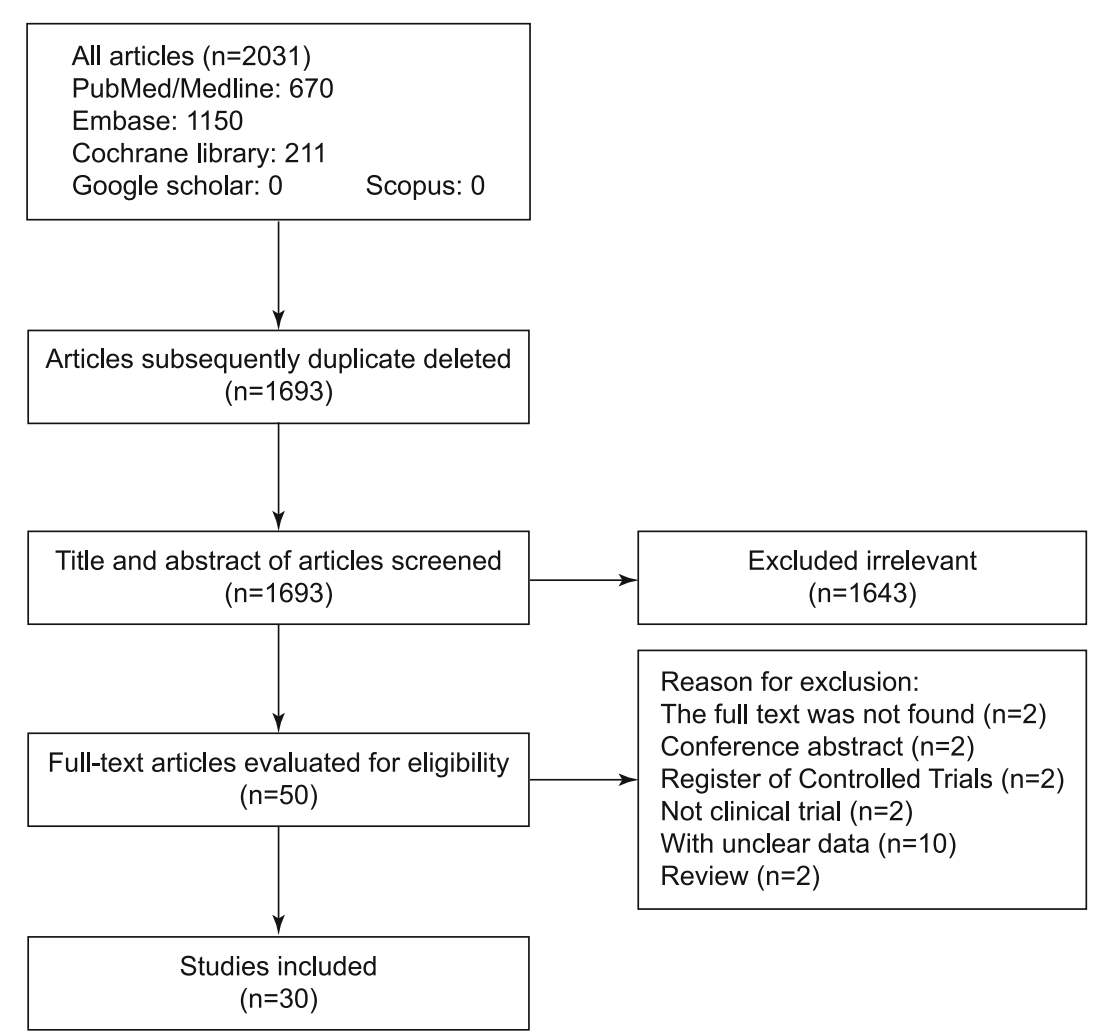

Fig. 1. Diagram of study selection for the inclusion in this research. 
Tab. 1. Features of the involved studies.

\begin{tabular}{|c|c|c|c|c|c|c|c|c|}
\hline First author & $\begin{array}{c}\text { Published } \\
\text { year }\end{array}$ & Country & $\begin{array}{l}\text { Study } \\
\text { design }\end{array}$ & $\begin{array}{c}\text { Mean } \\
\text { Age (yrs.) }\end{array}$ & $\begin{array}{l}\text { Total No. of } \\
\text { Patients } \\
\text { Enrolled } \\
\end{array}$ & $\begin{array}{l}\text { Follow-up } \\
\text { (mo.) }\end{array}$ & $\begin{array}{l}\text { Time from PCI } \\
\text { and/or MI to } \\
\text { transplantation }\end{array}$ & Cell type \\
\hline Laguna (15) & 2018 & Spain & RCT & 62.63 & 20 & 9 & 10-15 days & BMMNC \\
\hline Duan (16) & 2015 & China & RCT & 57.88 & 42 & 12 & NR & BMMNC \\
\hline Gao (17) & 2015 & China & RCT & 57.3 & 116 & 18 & 6.3 days & WJMSCs \\
\hline $\mathrm{Hu}(\mathrm{N})(18)$ & 2015 & China & RCT & 61.2 & 25 & 12 & $3-5$ days & N-BMCs \\
\hline Hu (HP) (18) & 2015 & China & RCT & 59.7 & 25 & 12 & 3-5 days & HP-BMCs \\
\hline Duan F (19) & 2015 & China & RCT & 57.88 & 42 & 12 & NR & BMC \\
\hline Chullikana (20) & 2014 & India & RCT & 47.31 & 20 & 24 & 2 days & BMMSC \\
\hline Lee j (21) & 2014 & USA & RCT & 53.9 & 48 & 6 & NR & MSCs \\
\hline Gao (22) & 2013 & China & RCT & 55 & 43 & 12 & 17.1 days & BMSCs \\
\hline Moccetti (23) & 2012 & Switzerland & RCT & 55 & 60 & 60 & 3 days & BMC \\
\hline Traverse (24) & 2010 & USA & RCT & 52.5 & 40 & 6 & 4.5 days & BMC \\
\hline Grajek (25) & 2010 & Poland & RCT & 49.9 & 45 & 12 & 4-6 days & BMSCs \\
\hline Beitnes (26) & 2009 & Norway & RCT & 58.1 & 100 & 36 & 4-8 days & $\mathrm{BMC}$ \\
\hline Herbots (27) & 2009 & Belgium & RCT & 55 & 67 & 4 & NR & BMPCs \\
\hline Plewka (28) & 2009 & Poland & RCT & 56 & 60 & 6 & 7 days & BMSC \\
\hline Huikuri (29) & 2008 & Finland & RCT & 60 & 80 & 6 & 2-6 days & $\mathrm{BMC}$ \\
\hline Yаo (30) & 2008 & China & RCT & 54.8 & 47 & 6 & 13 months & $\mathrm{BMC}$ \\
\hline Panovsky (HD) (31) & 2008 & Czech Republic & RCT & 55 & 34 & 3 & 5-9 days & $\mathrm{BMC}$ \\
\hline Panovsky (LD) (31) & 2008 & Czech Republic & RCT & 55 & 30 & 3 & 5-9 days & $\mathrm{BMC}$ \\
\hline Meluzín-2 (31) & 2007 & Czech Republic & RCT & 48 & 12 & 6 & 5-9 days & $\mathrm{BMC}$ \\
\hline Choi (32) & 2007 & Korea & NR & 50.5 & 33 & 6 & 6 days & PBSCs \\
\hline Zhan-quan (33) & 2007 & China & NR & 60 & 58 & 6 & NR & PBSCs \\
\hline Penicka (Horak) (34) & 2007 & Czech Republic & RCT & 59 & 27 & 4 & NR & BMNCs \\
\hline Ge j (Qian) (35) & 2006 & China & RCT & 58 & 20 & 6 & $12 \mathrm{~h}$ & $\mathrm{BMC}$ \\
\hline Meluzín-1 (LD) (36) & 2006 & Czech Republic & RCT & 44 & 55 & 3 & 7 days & $\mathrm{BMC}$ \\
\hline Meluzín-1 (HD) (36) & 2006 & Czech Republic & RCT & 44 & 55 & 3 & 7 days & $\mathrm{BMC}$ \\
\hline Hendrikx (37) & 2006 & Belgium & RCT & 63.2 & 20 & 4 & $217 \pm 162$ days & BMC \\
\hline Janssens (38) & 2006 & Belgium & RCT & 55.8 & 67 & 4 & NR & BMSC \\
\hline Kang (39) & 2005 & Korea & RCT & 66.6 & 50 & 6 & NR & PBSCs \\
\hline Kang (39) & 2005 & Korea & RCT & 59.8 & 36 & 6 & NR & PBSCs \\
\hline Bartunek (40) & 2005 & Belgium & RCT & 51 & 38 & 4 & 11.6 days & CD133+ cells \\
\hline W Ruan (41) & 2005 & China & RCT & 61 & 20 & 6 & NR & BMC \\
\hline Chen (42) & 2004 & China & RCT & 58 & 69 & 3 & 18.4 days & BMSCs \\
\hline Wollert (43) & 2004 & Germany & RCT & 53.4 & 60 & 6 & 4.8 days & BMC \\
\hline
\end{tabular}

\section{Data extraction}

The following data were obtained: patient information, type of stem cells, follow-up durations, outcomes, study quality. Altering in LVEF, LVESV from baseline, and LVEDV were the primary outcomes. Two authors independently conducted the steps of the systematic review. Any disagreements were discussed and resolved between reviewers.

\section{Quality assessment}

Main assessment checklist for RCTs providing by the Joanna Briggs Institute (JBI) were utilized to determine the included studies quality (13).

\section{Data analysis}

Statistical analyses were done by comprehensive CMAS. Fixed or random-effect model were used to investigate the WMD with 95\% CI. Assessing the heterogeneity between the studies were done by the Cochran's Q and the I2 statistic. The I2 values more than $50 \%$ were calculated to show a significant level of heterogeneity (14). Egger's and Begg's tests (BET) were used to obtain publication bias. p value $<0.05$ for publication bias and funnel plots was considered significant.

\section{Results}

The studies included and excluded through the review process are summarized in Figure 1. In the initial search, totally 2031 records were found and then repetitious articles were deleted. Then, after screening the titles and abstracts of 1693 references, 50 papers were selected for a full-text assessment in which 30 studies (34 datasheets) met the inclusion criteria for the meta-analysis. As shown in Table 1, the studies which were conducted from 2004 to 2018, mostly in China (12 studies), with the patients mean age 55 years (from 44 to 66 old), the mean follow-up duration 9.6 months, 
Tab. S2. Quality of studies used in research.

\begin{tabular}{|c|c|c|c|c|c|c|c|c|c|c|c|c|c|}
\hline 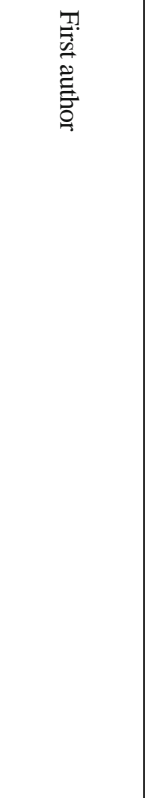 & 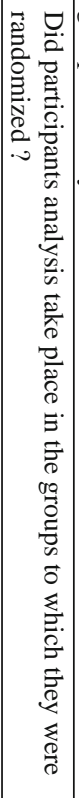 & 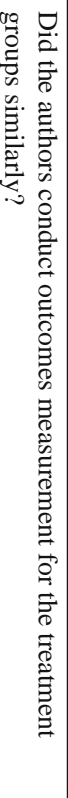 & 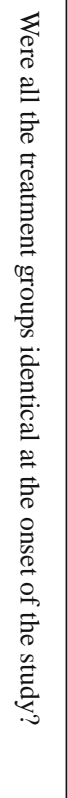 & 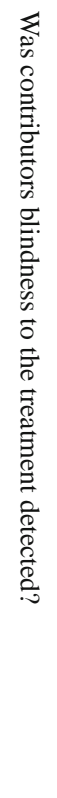 & 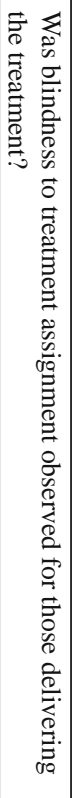 & 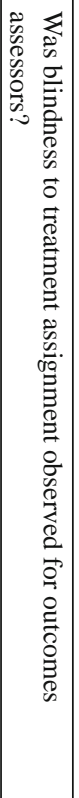 & 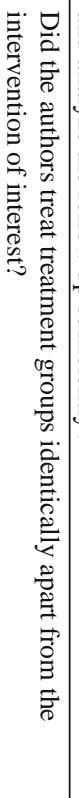 & 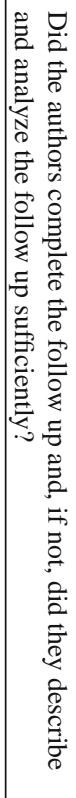 & $\begin{array}{l}\bar{a} \\
0 \\
0 \\
0 \\
0 \\
0 \\
0 \\
0 \\
0 \\
0 \\
0 \\
0 \\
0 \\
00 \\
0 \\
0 \\
0 \\
0 \\
0 \\
0 \\
0 \\
0 \\
0 \\
0 \\
0 \\
0 \\
0 \\
0 \\
0 \\
0 \\
00 \\
0 \\
0 \\
0 \\
0 \\
0 \\
0 \\
0 \\
0 \\
0 \\
.0\end{array}$ & 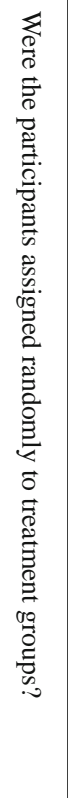 & 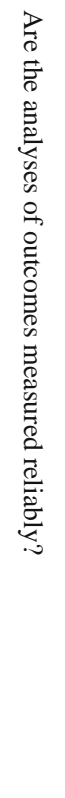 & 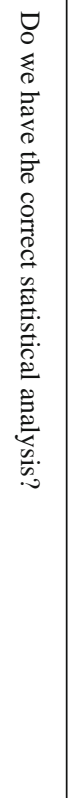 & 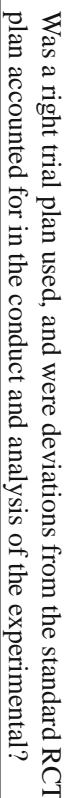 \\
\hline & & & Yes & Yes & es & Yes & $\mathrm{Y}$ & Yes & res & & 10 & Yes & \\
\hline & es & & Yes & Yes & Yes & Yes & Yes & Yes & Yes & Yes & Yes & Yes & Yes \\
\hline Sunc & es & es & Yes & Yes & Yes & Yes & Yes & Yes & Yes & $\mathrm{Ye}$ & 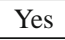 & Yes & Yes \\
\hline & ss & es & Yes & No & No & Yes & Yes & Yes & Yes & Yes & Yes & Yes & Yes \\
\hline & es & $Y$ & Yes & Yes & Yes & Yes & es & Yes & Yes & $\mathrm{Ye}$ & & IE & Yes \\
\hline & ss & es & Yes & Yes & Yes & Yes & Yes & Yes & Yes & Yes & Yes & Yes & Yes \\
\hline & ss & & Yes & Yes & es & Yes & & Yes & Yes & $\mathrm{Ye}$ & & & Yes \\
\hline $\mathrm{I}$ & Tes & & Yes & Yes & Yes & Yes & Yes & Yes & Yes & Yes & Yes & Yes & Yes \\
\hline & ss & & Yes & Tes & es & Yes & & Yes & Yes & $\mathrm{Ye}$ & & & Yes \\
\hline $\mathrm{Tr}$ & es & & Yes & Yes & Yes & Yes & Yes & Yes & Yes & Yes & Yes & Yes & Yes \\
\hline & & & Yes & & es & Yes & & Yes & Yes & $\mathrm{Ye}$ & & & Yes \\
\hline & s & & Yes & Kes & Yes & Yes & Yes & Yes & Yes & Yes & Yes & Yes & Yes \\
\hline & & & Yes & & & Yes & & Yes & & & & & Yes \\
\hline & es & & Yes & Tes & Yes & Yes & Yes & Yes & Yes & Yes & Yes & Yes & Yes \\
\hline & & & & & & es & & $\mathrm{Ye}$ & & & & & Yes \\
\hline & es & & Yes & Yes & Yes & Yes & Yes & Yes & Yes & Yes & Yes & Yes & Yes \\
\hline & & & Yes & Yes & & Yes & & Yes & & & & & Yes \\
\hline & es & & Yes & $?$ & Yes & Yes & Yes & Yes & Yes & Yes & Yes & Yes & Yes \\
\hline & & & & & & Yes & & Yes & & & & & Yes \\
\hline & es & & Yes & Yes & Yes & Yes & Yes & Yes & Yes & Yes & Yes & Yes & Yes \\
\hline & & & & Yes & Yes & es & & Yes & $1 \mathrm{~s}$ & & & & Yes \\
\hline & es & & Yes & $?$ & $?$ & Yes & Yes & Yes & Yes & Yes & Yes & Yes & Yes \\
\hline & & & & Yes & Yes & es & & Yes & 103 & & & & Yes \\
\hline Ple & es & & Yes & $?$ & 3 & Yes & Yes & Yes & Yes & Yes & Yes & Yes & Yes \\
\hline & & & & Yes & yes & Yes & & Yes & Yes & Yes & & Tes & Yes \\
\hline & & & Yes & ? & $?$ & Yes & Yes & Yes & Yes & Yes & Yes & Yes & Yes \\
\hline & es & & Yes & tes & yes & Yes & es & Yes & Yes & Yes & Yes & Yes & Yes \\
\hline Grajek & es & & Yes & Yes & yes & Yes & Yes & Yes & Yes & Yes & Yes & Yes & Yes \\
\hline Janssens & es & & Yes & Yes & yes & Yes & Yes & Yes & Yes & Yes & Yes & Yes & Yes \\
\hline Moccetti & es & & Yes & Yes & yes & Yes & Yes & Yes & Yes & Yes & Yes & Yes & Yes \\
\hline Schaching & Tes & & Yes & Yes & yes & Yes & Yes & Yes & Yes & Yes & Yes & Yes & Yes \\
\hline Wen & Yes & & Yes & Yes & yes & Yes & Yes & Yes & Yes & Yes & & Yes & Yes \\
\hline Qi & Yes & Yes & Yes & Yes & yes & Yes & Yes & Yes & Yes & Yes & Yes & Yes & Yes \\
\hline 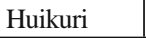 & Yes & Yes & Yes & Yes & yes & Yes & Yes & Yes & Yes & Yes & Yes & Yes & Yes \\
\hline
\end{tabular}

patient total numbers 1617 , and studies sample size range from 12 to 116 were selected. Notably, included studies had a low risk of bias according to the JBI tool which is shown in Table S2. Criteria were included: (1) the intervention contained of any stem cells without limitation via administration route or dose, (2) at least three months' follow-up period, and (3) in the control arm patients did not receive stem cells.

Effect of stem cell therapy on changes in LVEF, LVEDV, and LVESV after MI

A total of 29 RCTs (33 datasheets) with 1458 participants assessed the relationship between the stem cell therapy and changes in LVEF in patients after MI. In the overall pooled estimate, stem cell therapy has the capacity to change LVEF from baseline to follow-up (WMD: $2.98 \mathrm{~mL}, 95 \% \mathrm{CI}$ : 1.66 to $4.29 \mathrm{~mL}, \mathrm{p}=0.00$, I2 = 91 \%; (Fig. 2). Stem cell therapy and LVEDV changes interconnection was also investigated by 25 RCTs (29 datasheets) with 1324 participants. The pooled WMD was found to be $-4.16 \mathrm{~mL}$, $95 \% \mathrm{CI}:-7.91$ to $-0.40 \mathrm{~mL}, \mathrm{P}=0.03$, I2 = $78 \%$ (Fig. 3) and thus the participants in the treatment group experienced a significant lowering in LVEDV. As well as in an overall pooled estimate, which included a total of 24 trials (27 datasheets) with 1243 participants, the patients had a significantly decrease in the cell therapy group compared to the control group in LVESV from the reference point to follow-up (WMD:-5.62 mL, 95\% CI:-9.20 to $-2.00 \mathrm{~mL}, \mathrm{p}=0.00, \mathrm{I}=85 \%$ ) (Fig. 4).

\section{Publication bias}

A statistical analysis of funnel plots (Figs S1-S3) suggested no publication bias BET, p > 0.05 for LVEF, LVEDV, and LVESV).

\section{Discussion}

Current study assessed the safety and ability of stem cell therapy in MI through a systematic review on the RCT studies. Although there were some contradictions on the stem cells transplantation usefulness, the result of this study is consistent with those studies indicating that stem cell therapy is an effective treatment in people, who experienced MI $(45,46)$.

As mentioned before, the focus of this study was on those RCT studies with the 


\begin{tabular}{|c|c|c|c|}
\hline \multirow[t]{2}{*}{ Study name } & \multicolumn{3}{|c|}{ Statistics for each study } \\
\hline & $\begin{array}{c}\text { Difference } \\
\text { in means }\end{array}$ & $\begin{array}{c}\text { Lower } \\
\text { limit }\end{array}$ & $\begin{array}{c}\text { Upper } \\
\text { limit }\end{array}$ \\
\hline Chullikana & 1.960 & -6.060 & 9.980 \\
\hline Laguna & 1.420 & -5.448 & 8.288 \\
\hline Huikuri & 3.000 & -2.620 & 8.620 \\
\hline Kang (AMI) & 4.000 & -1.653 & 9.653 \\
\hline Kang (OMI) & 3.200 & -5.033 & 11.433 \\
\hline Qian & 2.300 & -4.208 & 8.808 \\
\hline Beitnes & 0.700 & -2.803 & 4.203 \\
\hline Meluzin-1 (LD) & 1.000 & -0.182 & 2.182 \\
\hline Meluzin-1 (HD) & 2.000 & 0.818 & 3.182 \\
\hline Meluzin-2 & 1.000 & -4.123 & 6.123 \\
\hline Traverse & -1.800 & -9.502 & 5.902 \\
\hline Choi & -2.600 & -9.759 & 4.559 \\
\hline Bartunek & 3.500 & 1.242 & 5.758 \\
\hline Lee & 4.600 & -0.916 & 10.116 \\
\hline Yao & 2.200 & -1.832 & 6.232 \\
\hline Wollert & 4.700 & -1.601 & 11.001 \\
\hline Zhan-quan & 4.500 & 0.791 & 8.209 \\
\hline Gao-2013 & 0.700 & -0.305 & 1.705 \\
\hline Gao-2015 & 6.000 & 5.754 & 6.246 \\
\hline Herbots & 1.400 & -2.291 & 5.091 \\
\hline Hendrikx & 5.800 & -3.162 & 14.762 \\
\hline Horak & -2.000 & -8.687 & 4.687 \\
\hline Plewka & 6.000 & 0.862 & 11.138 \\
\hline Panovsky (HD) & 2.000 & 0.655 & 3.345 \\
\hline Panovsky (LD) & 2.000 & 0.556 & 3.444 \\
\hline Chen & 13.000 & 11.047 & 14.953 \\
\hline Grajek & 2.550 & -3.276 & 8.376 \\
\hline Janssens & 2.700 & -2.257 & 7.657 \\
\hline Moccetti & 4.000 & -1.933 & 9.933 \\
\hline Wen & 9.030 & -0.307 & 18.367 \\
\hline $\mathrm{Hu}(\mathrm{N})$ & -2.800 & -8.746 & 3.146 \\
\hline $\mathrm{Hu}(\mathrm{HP})$ & -3.500 & -10.053 & 3.053 \\
\hline Qi & 7.700 & 6.455 & 8.945 \\
\hline & 2.981 & 1.667 & 4.296 \\
\hline
\end{tabular}

Weight (Random)
Relative
weight
1.73
2.09
2.57
2.56
1.67
2.22
3.61
4.67
4.67
2.79
1.82
1.99
4.25
2.62
3.33
2.30
3.50
4.72
4.85
3.51
1.49
2.15
2.79
4.62
4.59
4.39
2.49
2.87
2.44
1.41
2.44
2.20
4.65
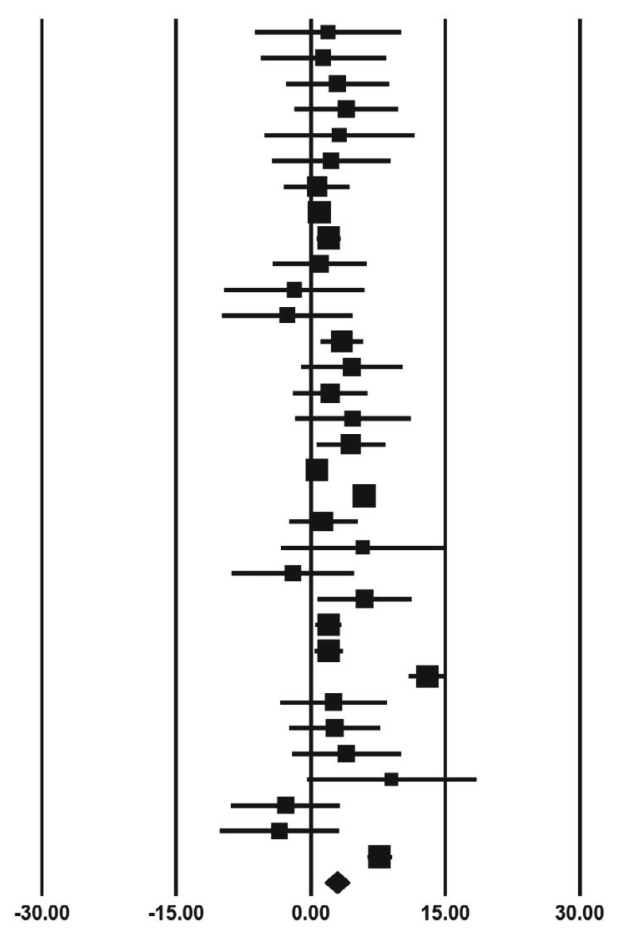

Fig. 2. Positive efficacy of stem cell therapy on changes in LVEF in post-MI cases compared to control.

\begin{tabular}{lrrr} 
Study name & & \multicolumn{3}{c}{ Statistics for each study } \\
\cline { 2 - 4 } & $\begin{array}{r}\text { Difference } \\
\text { in means }\end{array}$ & $\begin{array}{c}\text { Lower } \\
\text { limit }\end{array}$ & $\begin{array}{c}\text { Upper } \\
\text { limit }\end{array}$ \\
Duan & -11.400 & -35.208 & 12.408 \\
Laguna & 6.520 & -24.739 & 37.779 \\
Huikuri & -2.000 & -23.303 & 19.303 \\
Kang (AMI) & 6.600 & -13.330 & 26.530 \\
Kang (OMI) & 2.800 & -30.706 & 36.306 \\
Beitnes & -1.000 & -19.758 & 17.758 \\
Meluzin-1 (LD) & -14.000 & -20.527 & -7.473 \\
Meluzin-1 (HD) & -13.000 & -20.091 & -5.909 \\
Traverse & -10.000 & -24.278 & 4.278 \\
Choi & 16.000 & -0.085 & 32.085 \\
Bartunek & -4.000 & -9.127 & 1.127 \\
Lee & 7.200 & -5.987 & 20.387 \\
Yao & -2.000 & -5.665 & 1.665 \\
Wollert & 6.800 & -6.082 & 19.682 \\
Zhan-quan & 5.700 & -9.046 & 20.446 \\
Gao-2013 & -4.500 & -8.272 & -0.728 \\
Gao-2015 & -13.700 & -14.609 & -12.791 \\
Herbots & -7.000 & -24.393 & 10.393 \\
Hendrikx & -5.700 & -25.988 & 14.588 \\
Horak & -2.000 & -28.008 & 24.008 \\
Panovsky (HD) & -2.000 & -10.410 & 6.410 \\
Panovsky (LD) & -3.000 & -12.085 & 6.085 \\
Grajek & 9.570 & -21.600 & 40.740 \\
Janssens & -1.800 & -12.002 & 8.402 \\
Moccetti & -23.000 & -43.607 & -2.393 \\
Wen & -54.350 & -92.447 & -16.253 \\
Hu (N) & 17.500 & -21.620 & 56.620 \\
Hu (HP) & -1.200 & -31.903 & 29.503 \\
Qi & -11.400 & -35.208 & 12.408 \\
& -4.160 & -7.916 & -0.403
\end{tabular}

\section{Weight (Random)}

Relative weight

1.87
1.21
2.20
2.41
1.08
2.61
6.10
5.90
3.59
3.15
6.56
3.89
6.98
3.98
3.47
6.95
7.44
2.87
2.35
1.63
5.44
5.20
1.22
4.82
2.30
0.86
0.82
1.25
1.87

Difference in means and $95 \% \mathrm{Cl}$

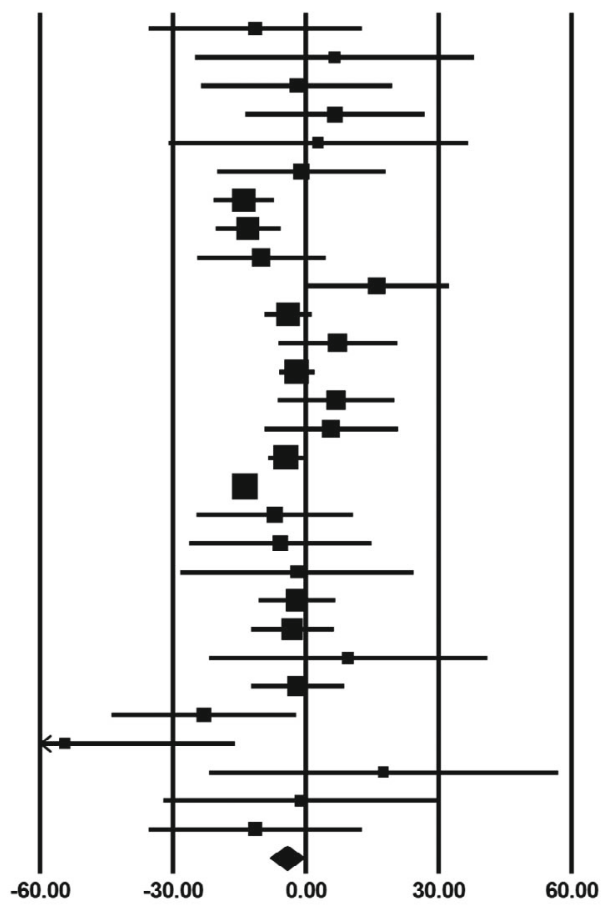

Fig. 3. Positive efficacy of stem cell therapy on changes in LVEDV in post-MI patients compared to control. 


\begin{tabular}{|c|c|c|c|c|c|}
\hline \multirow[t]{2}{*}{ Study name } & \multicolumn{3}{|c|}{$\underline{\text { Statistics for each study }}$} & \multirow{2}{*}{$\begin{array}{l}\text { Weight (Random) } \\
\begin{array}{l}\text { Relative } \\
\text { weight }\end{array}\end{array}$} & \multirow[t]{2}{*}{ Difference in means and $95 \% \mathrm{Cl}$} \\
\hline & $\begin{array}{l}\text { Difference } \\
\text { in means }\end{array}$ & $\begin{array}{c}\text { Lower } \\
\text { limit }\end{array}$ & $\begin{array}{c}\text { Upper } \\
\text { limit }\end{array}$ & & \\
\hline Duan & -13.520 & -33.047 & 6.007 & 2.23 & \\
\hline Laguna & -2.980 & -21.437 & 15.477 & 2.40 & \\
\hline Huikuri & -4.000 & -16.503 & 8.503 & 3.65 & \\
\hline Kang (AMI) & -5.700 & -22.588 & 11.188 & 2.67 & \\
\hline Kang (OMI) & -2.900 & -31.866 & 26.066 & 1.24 & \\
\hline Beitnes & -29.000 & -53.365 & -4.635 & 1.63 & \\
\hline Meluzin-1 (LD) & -11.000 & -16.351 & -5.649 & 5.74 & \\
\hline Meluzin-1 (HD) & -11.000 & -16.622 & -5.378 & 5.67 & \\
\hline Traverse & 1.000 & -3.351 & 5.351 & 6.00 & \\
\hline Choi & 8.500 & -5.842 & 22.842 & 3.20 & \\
\hline Lee & 4.300 & -5.059 & 13.659 & 4.54 & \\
\hline Yao & -0.400 & -4.939 & 4.139 & 5.96 & \\
\hline Wollert & -0.200 & -12.194 & 11.794 & 3.79 & \\
\hline Zhan-quan & 0.000 & -8.903 & 8.903 & 4.68 & \\
\hline Gao-2013 & -2.700 & -5.620 & 0.220 & 6.31 & \\
\hline Gao-2015 & -13.600 & -13.953 & -13.247 & 6.58 & \\
\hline Herbots & -6.000 & -16.134 & 4.134 & 4.31 & \\
\hline Hendrikx & -8.300 & -29.284 & 12.684 & 2.02 & \\
\hline Horak & -1.000 & -23.722 & 21.722 & 1.80 & \\
\hline Panovsky (HD) & -8.000 & -14.756 & -1.244 & 5.33 & \\
\hline Panovsky (LD) & -9.000 & -16.359 & -1.641 & 5.15 & \\
\hline Grajek & -2.970 & -18.499 & 12.559 & 2.94 & \\
\hline Janssens & -4.000 & -12.473 & 4.473 & 4.81 & \\
\hline Moccetti & -18.000 & -39.293 & 3.293 & 1.98 & \\
\hline Wen & -28.750 & -54.811 & -2.689 & 1.47 & \\
\hline $\mathrm{Hu}(\mathrm{N})$ & 4.500 & -19.538 & 28.538 & 1.66 & \\
\hline \multirow[t]{2}{*}{ Qi } & -13.520 & -33.047 & 6.007 & 2.23 & \\
\hline & -5.622 & -9.204 & -2.040 & & \\
\hline
\end{tabular}

Fig. 4. Positive efficacy of stem cell therapy on changes in LVESV in post-MI patients compared to control.

following criteria; using stem cells without a restriction on administration route or dose, at least three months follow up along with the control group without receiving stem cells. Our results demonstrated that stem cell therapy had a significant impact on the LVEF and improved it from the baseline to follow-up. As well, the WMD value for LVEDV, and LVESV significantly reduced after the stem cell therapy in the treatment group compared to the control group. Ischemia and MI as a pathophysiological stimulus leads to multiple molecular and cellular processes called ventricular remodeling that results in heart failure $(44,45)$. The most promising goal of using stem cells as a novel treatment is its potential to convert remodeling process to regeneration process. That is why, through cardiac remodeling, cardiomyocytes loss happened as the result of different cell death pathway like apoptosis, necrosis, and autophagy. Moreover, cardiomyocytes' shape and size are affected by mechanical and neuro-humoral triggers and build-up additional extracellular matrix (ECM) leads to fibrosis (42, 43, 46, 47). It has been indicated that the stem cell therapy has the capability to change remodeling process to regeneration process by stimulation of endogenous cardiac stem cells, stimulation of angiogenesis, reduction of myocardial fibrosis, restoration of contractile function, deleterious pathological remodeling, and can induce revascularization of the injured region (45, 47). Moreover, literature also indicates that the cellular mechanisms involving exosomes, connexin, mitochondrial transfer etc. Appear to have a significant role in the cardiac renovation $(45,47)$. As previously explained, three predictive values, including LVEF, LVEDV, and LVESV provide an outline to estimate the efficacy of the cell therapy. Based on our investigation, at least up to 3 months' follow-up is needed to observe the positive outcome of stem cell therapy on these markers. In this way, those patients with AMI with given stem cells, showed a significant reduction in the value of LVEDV, and LVESV. This result confirmed that cell therapy was effective in preserving LV contractility similar to the previous observations (48). This study was distinctive compared to the previous study in some way. First, previous investigation was done on observational (cohorts) and interventional (non-controlled and controlled clinical trials) studies, whereas our study was concerned with RCTs only. Second, their studies investigated treated patients with AMI and IHD, whereas our work was focused on MI. Third, their analysis was based on just 11 studies with a total

Funnel plot of standard error by difference in means

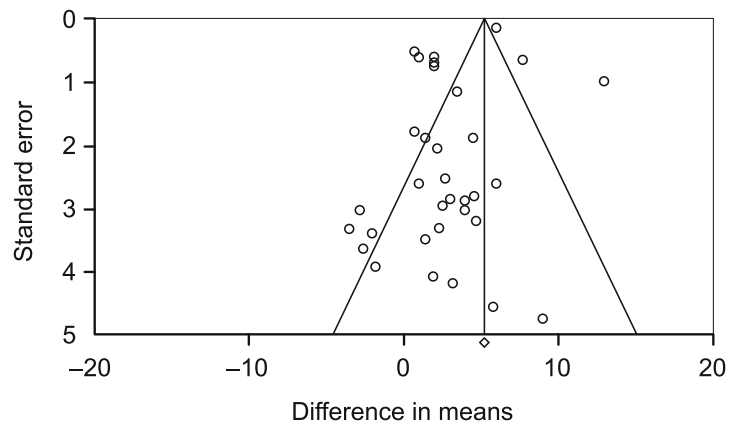

Fig. S1. Funnel plot of studies on LVEF. 
Funnel plot of standard error by difference in means

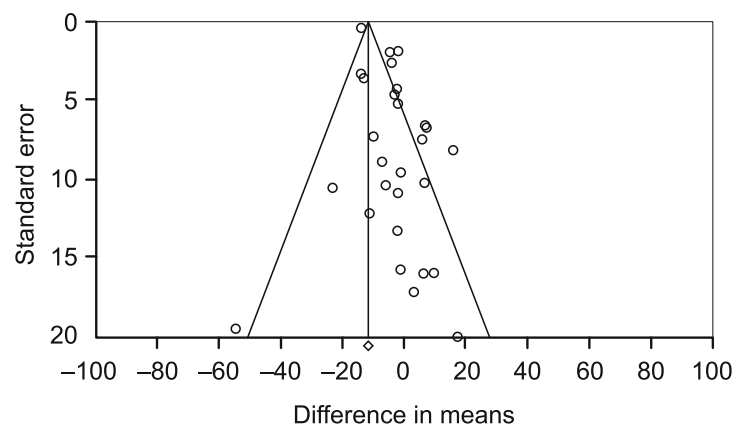

Fig. S2. Funnel plot of studies on LVEDV.

Funnel plot of standard error by difference in means

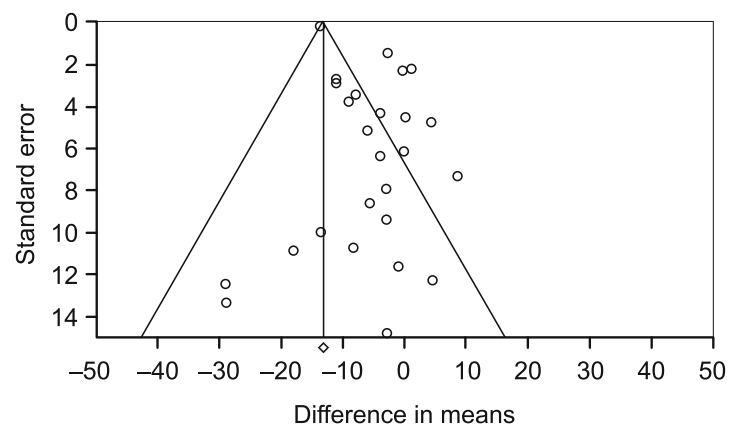

Fig. S3. Funnel plot of studies on LVESV.

of 509 participants. Even though, the current study described the efficacy of cell therapy, further investigation on a larger sample size to give more reliable results are required. Notably, this study has some limitations. First, an appreciable grade of heterogeneity was detected among the involved RCTs which might be the result of randomization nature, the timing between MI and cell therapy, the type and dose of cell infused, the infused cell quality, the methodology involved in the outcome measurements, and the baseline LVEF. Second, the analysis combined short-term with long-term outcomes as well as combined the patients with acute and chronic MI. Third, the potential influence of dose and cell injection timing could not be analyzed because of the limited information obtained from the RCTs; this might reduce the power of the conclusions (46).

Finally, important biomarkers have been identified using proteomics in connection with heart disease and effective drugs have been proposed for treatment that, along with cell therapy, can help patients recover and survive (49).

\section{Conclusion}

The present analysis provides a broad evaluation of the efficacy of cell therapy after MI. Though the outcomes of recent clinical trials showed a great deal of variety through restricted long-term follow-up, this analysis show that stem cell therapy maybe clinically pertinent and suggest a novel solution for the treatment of MI.

\section{References}

1. Khan MA, Hashim MJ, Mustafa $\mathbf{H}$ et al. Global Epidemiology of Ischemic Heart Disease: Results from the Global Burden of Disease Study. Cureus 2020; 12 (7).

2. Sonnenschein, E. and J.A. Brody.Effect of population aging on proportionate mortality from heart disease and cancer, US 2000-2050. The J of Gerontology Series B: Psychol Sci Social Sci 2005; 60 (2): 110-S112.

3. Racca V, Spezzaferri R, Modica M et al. Functioning and disability in ischaemic heart disease. Disability and rehabilitation 2010; 1: 32 (Sup1): 42-49.

4. Chen K, Huang Y, Singh R, Wang ZZ. Arrhythmogenic risks of stem cell replacement therapy for cardiovascular diseases. J of cell phys 2020; 235 (9): 6257-6267.

5. Hashimoto H, Olson EN, Bassel-Duby R. Therapeutic approaches for cardiac regeneration and repair. Nat Rev Card 2018; 15 (10): 585-600.

6. Abbaszadeh HA, Niknazar S, Darabi S et al. Stem cell transplantation and functional recovery after spinal cord injury: a systematic review and meta-analysis. Anatom Cell Biol 2018; 51 (3): 180.

7. Darabi SH, Tiraihi T, Noori-Zadeh A et al. Creatine and retinoic acid effects on the induction of autophagy and differentiation of adipose tissuederived stem cells into GABAergic-like neurons. J Babol Uni Med Sci 2017; 19 (8): 41-9.

8. Peyvandi AA, Roozbahany NA, Peyvandi H et al. Critical role of SDF1/CXCR4 signaling pathway in stem cell homing in the deafened rat cochlea after acoustic trauma. Neu Reg Res 2018; 13 (1): 154.3.

9. Brucks S, Little WC, Chao T et al. Contribution of left ventricular diastolic dysfunction to heart failure regardless of ejection fraction. Amer $\mathrm{J}$ Cardiol 2005; 95 (5): 603-606.

10. McManus DD, Shah SJ, Fabi MR, Rosen A, Whooley MA, Schiller NB. Prognostic value of left ventricular end-systolic volume index as a predictor of heart failure hospitalization in stable coronary artery disease: data from the Heart and Soul Study. J Amer Soci Echo 2009; 1; 22 (2): 190-197.

11. Kato M, Kitada S, Kawada Y et al. Left Ventricular End-Systolic Volume Is a Reliable Predictor of New-Onset Heart Failure with Preserved Left Ventricular Ejection Fraction. Car Res Prac 2020; 29; 2020.

12. Moher D, Liberati A, Tetzlaff J, Altman DG. Preferred reporting items for systematic reviews and meta-analyses: the PRISMA statement. Int J Surg 2010; 1; 8 (5): 336-341.

13. Institute JB. The Joanna Briggs Institute critical appraisal tools for use in JBI systematic review: Checklist for Randomized Controlled Trials. 2019.

14. Higgins JP, Thompson SG. Quantifying heterogeneity in a meta-analysis. Stat Med 2002; 15; 21 (11): 1539-1558.

15. Laguna G, Maroto L, Fulquet $\mathbf{E}$ et al. Effect of direct intramyocardial autologous stem cell grafting in the sub-acute phase after myocardial infarction. J Cardiol Surg 2018; 1; 59 (2): 259-267.

16. Duan $\mathrm{F}$ et al. Effectiveness of bone marrow mononuclear cells delivered through a graft vessel for patients with previous myocardial infarction and chronic heart failure: an echocardiographic study of left ventricular remodeling. Med Ultrason 2015; 17 (2): 160-166.

17. Gao LR, Chen Y, Zhang NK et al. Intracoronary infusion of Wharton's jelly-derived mesenchymal stem cells in acute myocardial infarction: double-blind, randomized controlled trial. BMC Med 2015; 13 (1): 1-5.

18. Hu X, Huang X, Yang Q et al. Safety and efficacy of intracoronary hypoxia-preconditioned bone marrow mononuclear cell administration for acute myocardial infarction patients: The CHINA-AMI randomized controlled trial. Int J Cardiol 2015; 1; 184: 446-451. 
19. Duan F, Qi Z, Liu S et al. Effectiveness of bone marrow mononuclear cells delivered through a graft vessel for patients with previous myocardial infarction and chronic heart failure: an echocardiographic study of left ventricular remodeling. Med Ultra 2015; 1; 17 (2): 160-166.

20. Chullikana A, Majumdar AS, Gottipamula S et al. Randomized, double-blind, phase I/II study of intravenous allogeneic mesenchymal stromal cells in acute myocardial infarction. Cytotherapy 2015; 1; 17 (3): 250-261.

21. Lee JW, Lee SH, Youn YJ et al. A randomized, open-label, multicenter trial for the safety and efficacy of adult mesenchymal stem cells after acute myocardial infarction. J Kor Med Sci 2014; 29 (1): 23.

22. Gao LR, Pei XT, Ding QA et al. A critical challenge: dosage-related efficacy and acute complication intracoronary injection of autologous bone marrow mesenchymal stem cells in acute myocardial infarction. Int J Cardiol 2013; 9; 168 (4): 3191-3199.

23. Moccetti T, Sürder D, Klersy C et al. Sustained improvement in left ventricular function after bone marrow derived cell therapy in patients with acute ST elevation myocardial infarction. A 5-year follow-up from the Stem Cell Transplantation in Ischaemic Myocardium Study. Swi Med Week 2012; 142: 13632

24. Traverse JH, McKenna DH, Harvey K et al. Results of a phase 1, randomized, double-blind, placebo-controlled trial of bone marrow mononuclear stem cell administration in patients following ST-elevation myocardial infarction. Amer Heart J 2010; 1; 160 (3): 428-434.

25. Grajek S, Popiel M, Gil L et al. Influence of bone marrow stem cells on left ventricle perfusion and ejection fraction in patients with acute myocardial infarction of anterior wall: randomized clinical trial: Impact of bone marrow stem cell intracoronary infusion on improvement of microcirculation. Eur Heart J 2010; 1; 31 (6): 691-702.

26. Beitnes JO, Hopp E, Lunde K et al. Long-term results after intracoronary injection of autologous mononuclear bone marrow cells in acute myocardial infarction: the ASTAMI randomised, controlled study. Heart 2009; 15; 95 (24): 1983-1989.

27. Herbots L, D’hooge J, Eroglu E et al. Improved regional function after autologous bone marrow-derived stem cell transfer in patients with acute myocardial infarction: a randomized, double-blind strain rate imaging study. Eur Heart J 2009; 1; 30 (6): 662-670.

28. Plewka M, Krzemińska-Pakuła M, Lipiec P et al. Effect of intracoronary injection of mononuclear bone marrow stem cells on left ventricular function in patients with acute myocardial infarction. Amer J Cardiol 2009; 15; 104 (10): 1336-1342.

29. Huikuri HV, Kervinen K, Niemelä M et al. Effects of intracoronary injection of mononuclear bone marrow cells on left ventricular function, arrhythmia risk profile, and restenosis after thrombolytic therapy of acute myocardial infarction. Eur Heart J 2008; 1; 29 (22): 2723-2732.

30. Yao K, Huang R, Qian J et al. Administration of intracoronary bone marrow mononuclear cells on chronic myocardial infarction improves diastolic function. Heart 2008; 1; 94 (9): 1147-1153.

31. Panovsky R, Meluzin J, Janousek $S$ et al. Cell therapy in patients with left ventricular dysfunction due to myocardial infarction. Echocardiography 2008; 25 (8): 888-897.

32. Choi JH, Choi J, Lee WS et al. Lack of additional benefit of intracoronary transplantation of autologous peripheral blood stem cell in patients with acute myocardial infarction. Circulat J 2007; 71 (4): 486-494.

33. Zhan-quan L, Ming Z, Yuan-zhe $\mathbf{J}$ et al. The clinical study of autologous peripheral blood stem cell transplantation by intracoronory infusion in patients with acute myocardial infarction (AMI). Int J Cardiol 2007; 31; 115 (1): 52-56
34. Penicka M, Horak J, Kobylka P et al. Intracoronary injection of autologous bone marrow-derived mononuclear cells in patients with large anterior acute myocardial infarction: a prematurely terminated randomized study. J Amer Coll Cardiol 2007; 19; 49 (24): 2373-2374.

35. Ge J, Li Y, Qian J, Shi J et al. Efficacy of emergent transcatheter transplantation of stem cells for treatment of acute myocardial infarction (TCTSTAMI). Heart 2006; 1; 92 (12): 1764-1767.

36. Meluzín J, Mayer J, Groch L et al. Autologous transplantation of mononuclear bone marrow cells in patients with acute myocardial infarction: the effect of the dose of transplanted cells on myocardial function. Amer Heart J 2006; 1; 152 (5): 975-e9.

37. Hendrikx M, Hensen K, Clijsters C et al. Recovery of regional but not global contractile function by the direct intramyocardial autologous bone marrow transplantation: results from a randomized controlled clinical trial. Circulation 2006; 114 (Suppl 1): I-101.

38. Janssens $\mathbf{S}$, Dubois $\mathbf{C}$, Bogaert J et al. Autologous bone marrow-derived stem-cell transfer in patients with ST-segment elevation myocardial infarction: double-blind, randomised controlled trial. Lancet 2006; 14; 367 (9505): 113-121.

39. Kang HJ, Lee HY, Na SH et al. Differential effect of intracoronary infusion of mobilized peripheral blood stem cells by granulocyte colony-stimulating factor on left ventricular function and remodeling in patients with acute myocardial infarction versus old myocardial infarction: The MAGIC cell-3DES randomized, controlled trial. Circulation 2006; 4; 114 (Suppl 1): I-145.

40. Bartunek J, Vanderheyden M, Vandekerckhove B et al. Intracoronary injection of CD133-positive enriched bone marrow progenitor cells promotes cardiac recovery after recent myocardial infarction: feasibility and safety. Circulation 2005; 30; 112 (Suppl 9): I-178.

41. Ruan W, Pan CZ, Huang GQ, Li YL, Ge JB, Shu XH. Assessment of left ventricular segmental function after autologous bone marrow stem cells transplantation in patients with acute myocardial infarction by tissue tracking and strain imaging. Chi Med J 2005; 1; 118 (14): 1175-1181.

42. Chen SL, Fang WW, Ye F et al. Effect on left ventricular function of intracoronary transplantation of autologous bone marrow mesenchymal stem cell in patients with acute myocardial infarction. Amer J Cardiol 2004; 1; 94 (1): 92-95.

43. Wollert KC, Meyer GP, Lotz J et al. Intracoronary autologous bonemarrow cell transfer after myocardial infarction: the BOOST randomised controlled clinical trial. Lancet 2004; l 10; 364 (9429): 141-148.

44. Wang Z, Wang L, Su X, Pu J, Jiang M, He B. Rational transplant timing and dose of mesenchymal stromal cells in patients with acute myocardial infarction: a meta-analysis of randomized controlled trials. Stem Cell Res Ther 2017; 8 (1): 1-0.

45. Lalu MM, Mazzarello S, Zlepnig $\mathbf{J}$ et al. Safety and efficacy of adult stem cell therapy for acute myocardial infarction and ischemic heart failure (SafeCell Heart): A systematic review and meta-analysis. Stem Cells Tra Med 2018; 7 (12): 857-866.

46. Karantalis V, Hare JM. Use of mesenchymal stem cells for therapy of cardiac disease. Circulat Res 2015; 116 (8): 1413-1430.

47. Wollert KC, Drexler H. Clinical applications of stem cells for the heart. Circulation res 2005; 96 (2): p. 151-163.

48. Martin-Rendon E, Brunskill SJ, Hyde CJ, Stanworth SJ, Mathur A, Watt SM. Autologous bone marrow stem cells to treat acute myocardial infarction: a systematic review. Eur Heart J 2008; 1; 29 (15): 1807-1818.

49. Amiri-Dashatan N, Koushki M, Abbaszadeh HA et al. Proteomics applications in health: biomarker and drug discovery and food industry. Iran J Pharm Res 2018; 17 (4): 1523.

Received April 20, 2021. Accepted May 4, 2021. 